\title{
AVERAGE VARIATIONS OF PHOTOSPHERIC FEI AND FEII LINE PARAMETERS AS FUNCTION OF THE MAGNETIC FILLING FACTOR *
}

\author{
P.N. BRANDT ${ }^{1}$ and M. STEINEGGER ${ }^{2}$ \\ ${ }^{1}$ Kiepenheuer-Institut für Sonnenphysik \\ Schöneckstr. 6 \\ D-7800 FREIBURG, F.R.G. \\ 2 Insitut für Astronomie \\ Universitatsplatz 5 \\ A-8010 GRAZ, Austria
}

\begin{abstract}
A series of 17 Fourier transform spectra taken at the McMath telescope near disk center in regions of different magnetic field strengths were analyzed. Applying a multi-variate regression analysis magnetic filling factors $0<\alpha \leq 0.11$ were determined. With $\alpha$ increasing from 0 to 0.11 , line bisectors averaged over groups of lines of similar depth are found to show a blue shift decreasing from $0.35 \mathrm{~km} \mathrm{~s}^{-1}$ to nearly $0.1 \mathrm{~km} \mathrm{~s}^{-1}$, when referred to the $\mathrm{MgI}$ line $\lambda 5172.7 \AA$. The bisectors of FeII lines exhibit smaller blue shifts than FeI lines. The increase of bisector red shift near the continuum with increasing $\alpha$, found earlier by Brandt and Solanki (1987), was confirmed and is tentatively interpreted as a manifestation of downdrafts in the vicinity of flux tubes (Deinzer et al., 1984).

A significant increase of line width (typically between 3 and $8 \%$, depending on line strength) and a decrease of line depth is found with increasing filling factor. For strong lines the equivalent width $\mathrm{W}$ shows no variation or a slight increase, while for the weaker lines a reduction of $\mathrm{W}$ between a few $\%$ and $>10 \%$ is found.
\end{abstract}

\section{Introduction}

The asymmetries and wavelength shifts of solar line profiles have been known for over 30 years (e.g. Schröter, 1957; for a summary cf. Dravins, 1982). They are usually attributed to the brightness-velocity correlation of the granulation. The " $\mathrm{C}$ "-shaped line asymmetry can be observed in spectrograms of high spatial resolution (Keil and Yackovich, 1981; Kavetsky and O'Mara, 1984; Mattig et al., 1989) as well as - to a lesser degree - in spectra representing spatial averages (e.g. Adam et al., 1976; Dravins et al., 1981; Brandt and Schröter, 1982). The Sun observed "as a star" also exhibits this line asymmetry (Livingston, 1982) and thus provides a "Rosetta stone" for the indirect observation of surface convection on stars (cf. Gray, 1982; Dravins, 1987a, 1987b).

The influence of magnetic fields on solar and stellar convection is of basic astrophysical interest and has been studied theoretically (cf. reviews by e.g. Nordlund, 1986; Hughes and Proctor, 1988) and observationally (e.g. Mattig and Nesis, 1976; Livingston, 1982, 1983; Kaisig and Schröter, 1983; Brandt and Schröter, 1984; Miller et al., 1984; Title et al., 1986, 1989; Immerschitt and Schröter, 1987, 1989; Cavallini et al., 1985, 1988; Brandt and Solanki, 1989). Many of these investigations are based on the difference of the " $\mathrm{C}$ " shape and wavelength shift between quiet and active regions

* Mitteilungen aus dem Kiepenheuer-Institut No. 311

41

J. O. Stenflo (ed.), Solar Photosphere: Structure, Convection, and Magnetic Fields, 41-46.

(c) 1990 by the IAU. 
on the Sun ('plages') and show as a result some straightening of the line bisectors and a slightly reduced blue shift in active regions which is interpreted as an indication of reduced dynamics in these areas. We present an analysis of Fourier transform spectrometer observations, which comprises a quantitative determination of the magnetic filling factors of the observed regions as well as a treatment of a large set of FeI and FeII lines.

\section{Observations and Data Evaluation}

For the analysis a set of 17 Fourier transform spectra were selected. They were observed in June 1984 at the McMath solar telescope in regions of varying magnetic activity and quiet Sun regions near disk center $(\cos \theta \geq 0.95)$ with a slit of 5 by $25 \operatorname{arcsec}^{2}$. The observed wavelength range was $\lambda \lambda 5050-6650 \AA$ with a resolution of 180000 and a $\mathrm{S} / \mathrm{N}$ ratio of 2000 to 3000 . An integration time of $13.7 \mathrm{~min}$ was used in order to average over several periods of the $5 \mathrm{~min}$ oscillation.

Continuum values were determined by parabolic fits through the highest intensity points in $75 \AA$ sections of the spectra and for ease of treatment the data were Fourier interpolated to wavenumber steps of $0.00656 \mathrm{~cm}^{-1}(3 \mathrm{~m} \AA-1.7 \mathrm{~m} \AA$ in wavelength). The magnetic filling factors $\alpha$ were estimated with the method developed by Stenflo and Lindegren (1977). This method is based on a multi-variate regression analysis of a line parameter (like line width or depth of the unpolarized profile) sensitive to Zeeman splitting. An average line weakening of 0.7 , a continuum contrast of 1.4 and a magnetic field strength of $1500 \mathrm{G}$ was assumed for the magnetic elements (for details $\mathrm{cf}$. Stenflo and Lindegren, 1977; Brandt and Solanki, 1987, 1989). Using 182 unblended FeI lines in each spectrum, magnetic filling factors $\alpha$ ranging from 0.00 to 0.11 were obtained for the 17 spectra.

For further analysis $187 \mathrm{FeI}$ lines and $23 \mathrm{FeII}$ lines were selected from the list given by Solanki and Stenflo (1985). They fulfilled the conditions of having no blends and line depths $\geq 0.1$. For each of these lines the position and residual intensity of the line minimum, the full width at half line depth 'FWHM', the equivalent width ' $W$ ' (by integration up to the nearest blend in the red and blue wing), and the bisector shape (between line minimum and $I / I_{c}=0.92$ ) were determined.

\section{Results}

\subsection{LINE BISECTORS: ASYMMETRIES AND SHIFTS}

For the specification of the shape of the line bisectors in the lower and upper part the following definitions were used: the wavelength difference $\left(\lambda_{\min }-\lambda_{0.7}\right)$ between line minimum and $I / I_{c}=0.7$ was denoted $\Delta \lambda_{\nabla}$, while correspondingly $\left(\lambda_{0.7}-\lambda_{0.9}\right)$ was denoted $\Delta \lambda_{\Delta}$. Fig. 1 shows a plot of these wavelength differences averaged over the group of 38 of the strongest $\mathrm{FeI}$ lines (line depths between 0.7 and $0.9 I / I_{c}$ ) as a function of the filling factor. The systematic decrease of $\Delta \lambda_{\nabla}$ in the lower part of the bisector and the increase from $-2.2 \mathrm{~m} \AA$ to $\approx-5 \mathrm{~m} \AA$ in the upper part can be seen clearly. The groups of the weaker lines show essentially the same behaviour somewhat less pronouncedly, due to the smaller range of heights of formation of these lines.

The wavelength positions of bisectors were determined with reference to the core of the MgI line $\lambda 5172.7 \AA$, which is supposed to show negligible granular blue shift (cf. Pierce and Breckinridge, 1973). The bisector positions were averaged in a two step process: $1^{\text {st }}$ groups of lines of similar depths were averaged in each spectrum, and $2^{\text {nd }}$ these averages were again averaged for spectra grouped according to increasing $\alpha$. The results are shown in Fig. 2a-c for two groups of FeI lines and one group of FeII lines.

For the strongest $\mathrm{FeI}$ lines a consistent decrease of the blue shift of the bisectors with increasing $\alpha$ from about $0.35 \mathrm{~km} \mathrm{~s}^{-1}$ at half the line depth to nearly $0.1 \mathrm{~km} \mathrm{~s}^{-1}$ for $\alpha$ between 0.04 and 0.08 with 


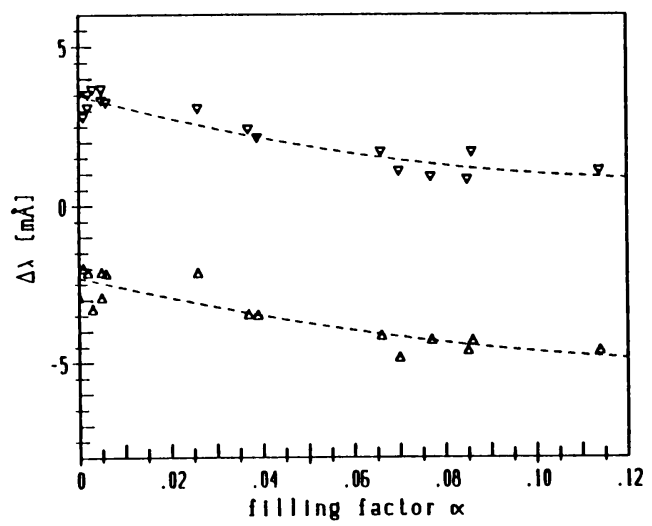

Fig.1: Wavelength differences in the lower part $\left(\nabla=\lambda_{\min }-\lambda_{0.7}\right)$ and upper part $\left(\Delta=\lambda_{0.7}-\lambda_{0.9}\right)$ of bisectors averaged over $38 \mathrm{FeI}$ lines of depths between 0.7 and $0.9 I / I_{c}$.
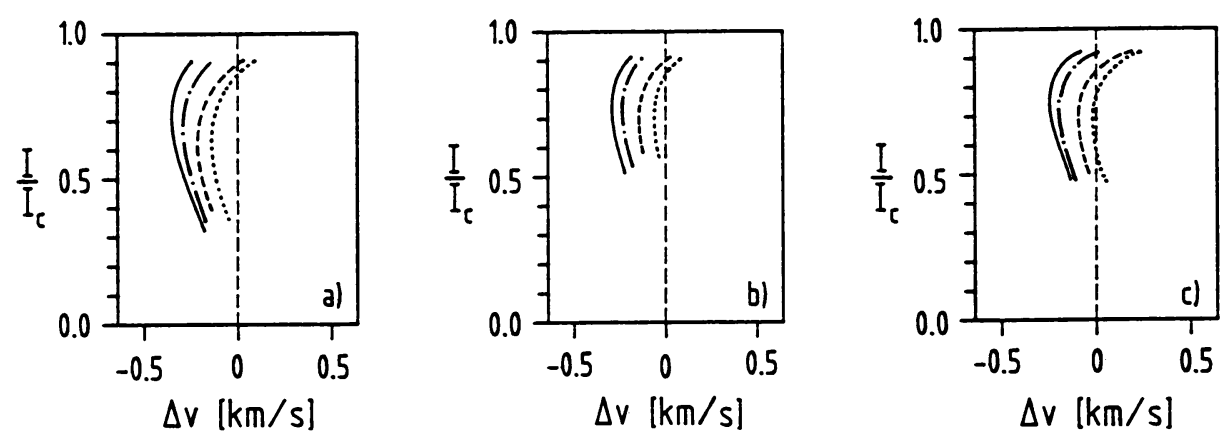

Fig. 2a-c: Average bisectors for groups of different line depths. a) $38 \mathrm{FeI}$ lines of $0.7<d \leq 0.9$; b) $73 \mathrm{FeI}$ lines of $0.5<\mathrm{d} \leq 0.7$; c) 3 FeII lines of $0.5<\mathrm{d} \leq 0.7$. Additionally the bisectors are averaged over several spectra grouped with increasing $\alpha$. Symbols: 8 spectra of $\alpha<0.01:-; 3$ spectra of $0.01 \leq \alpha<0.04$ : -.-..; 3 spectra of $0.04 \leq \alpha<0.08$ : ....; 3 spectra of $\alpha \geq 0.08:---$.

a slight increase for higher values of $\alpha$ is found. The FeII bisectors show blueshifts that are smaller by about $0.15 \mathrm{~km} \mathrm{~s}^{-1}$.

\subsection{LINE HALFWIDTHS}

In plots similar to Fig. 1 for each line second order polynomials were fitted into the variation of its FWHM with $\alpha$, and the relative change between $\alpha=0$ and $\alpha=0.11$ was determined. For the group of the 38 strongest FeI lines $(0.7<d<0.9)$ there is a consistent increase of the FWHM of 5 to $10 \%$. Here the Zeeman broadening was not subtracted. But this will be done in a future analysis. This is not mainly an effect of Zeeman broadening, which is demonstrated by the fact that the $3 \mathrm{FeI}$ lines with $\mathrm{g}=0$ ( $\lambda$ 5576.1 $\AA, \lambda 5434.5 \AA, \lambda 5123.7 \AA)$ show a broadening of between 4 and $6 \%$. The 
weaker lines increase their FWHM to a lesser extent: the group of $0.5<d<07$ between 3 and $8 \%$, the group of $0.3<d<0.5$ between 0 and $6 \%$, and the weakest lines between 0 and $5 \%$. Lines with excitation potential $\chi_{e}>3 \mathrm{eV}$ tend to exhibit smaller increases of the FWHM.

\subsection{LINE DEPTHS}

With $\alpha$ increasing from 0 to 0.11 all lines exhibit a significant decrease of the line depth, i.e. the lines become shallower. For the strongest lines $(0.7<d<0.9)$ this decrease ranges between 5 and $>10 \%$, and reaches values between 7 and $\approx 17 \%$ for the weaker lines. Here the lines of low excitation potential $\left(\chi_{e} \leq 3 \mathrm{eV}\right)$ show a significantly stronger decrease of $d$ than those of high $\chi_{e}$. The FeII lines consistently have a smaller decrease of line depth than the corresponding FeI lines of similar strength.

\subsection{EQUIVALENT WIDTHS}

Fig. 3a,b shows the variation of the equivalent width $W$ with $\alpha$ increasing from 0 to 0.11 as a function of the equivalent width itself. While the strongest lines show no change at all or a slight increase of $\mathrm{W}$, the medium-strong lines show a reduction between a few $\%$ and $10 \%$. The strongest decrease of $\mathrm{W}(\Delta \mathrm{W} / \mathrm{W} \approx 13 \%)$ is seen for the weakest lines, especially those of low excitation potential $\left(\chi_{e}<3\right.$ $\mathrm{eV})$.
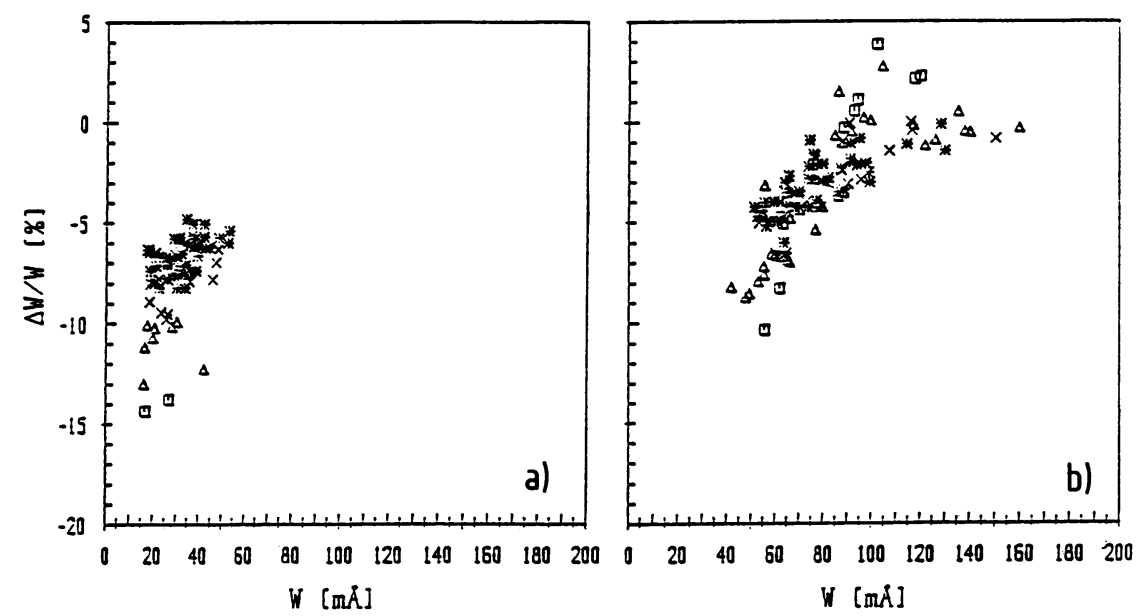

Fig. 3a,b: Changes of the equivalent widths between $\alpha=0$ and $\alpha=0.11$ as function of the equivalent width $W$ at $\alpha=0$. a) 76 lines of depth $d<0.5$; b) 111 lines of depth $\mathrm{d}>0.5$. Symbols: $\square \chi_{e} \leq 2 \mathrm{eV} ; \Delta 2<\chi_{e} \leq 3 \mathrm{eV} ; \times 3<\chi_{e} \leq 4 \mathrm{eV}$; $* \chi_{e}>4 \mathrm{eV}$.

\section{Discussion and Conclusion}

The gradually reduced blue shift of the line bisectors seen in active regions of increasing filling factor $\alpha$ can be interpreted in terms of a reduced vertical velocity component of the granular velocity field, possibly coupled to a modified intensity contrast. This would agree well with spectroscopic observations of reduced rms velocities in active regions (Nesis et al., 1989), which are indirectly 
confirmed by the observation of Title et al. $(1986,1989)$ of increased granular lifetimes and smaller horizontal proper motion velocities of granules in active regions, both facts hinting at reduced dynamics. On the other hand, since no net flow velocities in fluxtubes could be detected hitherto (Solanki, 1986), the increase of the redshift of the uppermost bisector part with increasing $\alpha$, already described by Brandt and Solanki (1987), may be due to increased downward velocities in the immediate vicinity of flux tubes, as postulated from model calculations by Deinzer et al. (1984). Also the broadening of most lines, especially of the $\mathrm{g}=0$ lines not affected by Zeeman broadening, in active regions seems to indicate increased "macro-turbulence", rather than a reduced velocity field. Only detailed model calculations, taking into consideration a realistic fluxtube model and a modified structure of the convection outside the fluxtubes can help to understand the riddle. The observations presented here try to establish reliable reference data to which the results of such model calculations can be compared.

\section{ACKNOWLEDGEMENTS}

The observations were carried out at the McMath solar telescope (N.O.A.O., Tucson). The assistance of J. Brault, B. Graves, R. Hubbard and G. Ladd is gratefully acknowledged. The National Optical Astronomy Observatories are operated by the Association of Universities for Research in Astronomy, Inc., under contract with the National Science Foundation. We are also indebted to S. Solanki for carrying out the filling factor analysis. One of us (P.N.B.) gratefully acknowledges financial support from the Deutsche Forschungsgemeinschaft.

\section{REFERENCES}

Adam, M.G., Ibbetson, P.A., Petford, A.D.: 1976, Mon. Not. Roy. Astron. Soc. 177, 687

Brandt, P.N., Schröter, E.-H.: 1982, Solar Phys. 79, 3

Brandt, P.N., Schröter, E.-H.: 1984, Small-Scale Dynamical Processes in Quiet Stellar Atmospheres, ed. S. Keil, NSO Conf., 371

Brandt, P.N., Solanki, S.K.: 1987, The Role of Fine-Scale Magnetic Fields on the Structure of the Solar Atmosphere, eds. E.-H. Schröter, M. Vazquez, A.A. Wyller, Cambridge Univ. Press, 82

Brandt, P.N., Solanki, S.K.: 1989, in preparation

Cavallini, F., Ceppatelli, G., Righini, A.: 1985, Astron. Astrophys. 143, 116

Cavallini, F., Ceppatelli, G., Righini, A.: 1988, Astron. Astrophys. 205, 278

Deinzer, W., Hensler, G., Schüssler, M., Weisshaar, E.: 1984, Astron. Astrophys. 139, 435

Dravins, D.: 1982, Ann. Rev. Astron. Astrophys. 20, 61

Dravins, D.: 1987a, Astron. Astrophys. 172, 200

Dravins, D.: 1987b, Astron. Astrophys. 172, 211

Dravins, D., Lindegren, L., Nordlund, Å.: 1981, Astron. Astrophys. 96, 345

Gray, D.F.: 1982, Astrophys. J. 255, 200

Hughes, D.W., Proctor, M.R.E.: 1988, Ann. Rev. Fluid Mech. 20, 187

Immerschitt, S., Schröter, E.-H.: 1987, The Role of Fine-Scale Magnetic Fields on the Structure of the Solar Atmosphere, eds. E.-H. Schröter, M. Vazquez, A.A. Wyller, Cambridge Univ. Press, 53

Immerschitt, S., Schröter, E.-H.: 1989, Astron. Astrophys. 208, 307

Kaisig, M., Schröter, E.-H.: 1983, Astron. Astrophys. 117305

Kavetsky, A., O'Mara, B.J.: 1984, Solar Phys. 92, 47

Keil, S.L., Yackovich, F.H.: 1981, Solar Phys. 69, 213

Livingston, W.C.: 1982, Nature 297, 208

Livingston, W.C.: 1983, Solar and Stellar Magnetic Fields: Origins and Coronal Effects, ed. J.O. Stenflo, IAU Symp. No. 102, 149 
Mattig, W., Nesis, A.: 1976, Solar Phys. 50, 255

Mattig, W., Hanslmeier, A., Nesis, A.: 1989, Solar and Stellar Granulation, Proc. NATO Adv. Res. Workshop, Capri, June 21-25, eds. R. Rutten and G. Severino, Kluwer, Dordrecht, 187

Miller, P., Foukal, P., Keil, S.: 1984, Solar Phys. 92, 33

Nesis, A., Fleig, K.-H., Mattig, W.: 1989, Solar and Stellar Granulation, Proc. NATO Adv. Res. Workshop, Capri, June 21-25, eds. R.J. Rutten and G. Severino, Kluwer, Dordrecht, 289 Nordlund, A.: 1986, Small Magnetic Flux Concentrations in the Solar Photosphere, eds.

W. Deinzer, M. Knölker, H.H. Voigt, Vandenbeck \& Ruprecht, Göttingen, 83

Pierce, A.K., Breckinridge, J.B.: 1973, Kitt Peak National Obs. Contr. No. 559

Schröter, E.-H.: 1957, Z. Astrophysik 41, 141

Solanki, S.K.: 1986, Astron. Astrophys. 168, 311

Solanki, S.K., Stenflo, J.O.: 1985, Astron. Astrophys. 148, 123

Stenflo, J.O., Lindegren, L.: 1977, Astron. Astrophys. 59, 367

Title, A.M., Tarbell, T.D., Simon, G.W., and the SOUP Team: 1986, Adv. Space Res. Vol. 6, No. 8, 253

Title, A.M., Tarbell, T.D., Topka, K.P., Ferguson, S.H., Shine, R.A., and the SOUP Team: 1989, Astrophys. J. 336, 475 